\title{
In vitro expression, characterisation and subcellular localisation of AvrPi54 protein, an effector of rice blast fungus
}

\section{Magnaporthe oryzae}

\author{
Banita K. Saklani ${ }^{1,2}$, Kirti Arora ${ }^{4}$, Ravi K. Asthana ${ }^{2}$, Tilak R. Sharma ${ }^{1,3}$ \\ ${ }^{1}$ ICAR-National Research Centre on Plant Biotechnology, New Delhi, India; \\ ${ }^{2}$ Banaras Hindu University, Varanasi, U.P., India; \\ ${ }^{3}$ National AgriFood Biotechnology Institute, Mohali, Punjab, India; ${ }^{4}$ Indian Agricultural \\ Research Institute, New Delhi, India. \\ * Corresponding author: Dr. T. R. Sharma; National Agri-Food Biotechnology Institute, \\ Mohali, Punjab, India; e-mail: trsharma@nabi.res.in; Phone 91-172-4990101/4011916
}

\begin{abstract}
Plant pathogens utilize effectors to subvert host cell biology for facilitating infection. However certain effectors, called as the avirulence proteins, trigger immune responses in the host. AvrPi54 is an avirulence protein from fungus Magnaporthe oryzae which induces the defense reactions in rice cells that contain Pi54 resistance gene. The characterization of such proteins and elucidation of their function facilitate the understanding of the mechanism of disease establishment. In present study, physiochemical properties of AvrPi54 were analysed using computational methods. We found that mature AvrPi54 is a small, hydrophobic and secretary protein with 134 amino acids. It is rich in small amino acids with no significant homology with other proteins in databases. The gene ORF was cloned in pET28a(+) vector and expressed in E. coli BL21(DE3)pLysS. The protein was purified using affinity chromatography. Transient expression of the gene in epidermal cells of onion bulb is a powerful tool to predict the subcellular localization in plant cells. We fused AvrPi54 to eYFP and transformed epidermal cell layer of onion bulb. Fused protein localized to the plasma membrane and nucleus of plant cells. Therefore, AvrPi54 might be functioning in the host cell as transcription factor or chromatin remodelling factor to modify pathogenesisrelated processes.
\end{abstract}

Keywords: avirulence; resistance; avrPi54; secretory protein; localization; particle bombardment 


\section{Introduction}

Plants are host to several pathogens and pests. These organisms derive niche and nutrition from the plant which is an unthrifty affair for the plant. Therefore, the plants have evolved many mechanisms to recognise a pathogen and avoid infection. Plants identify certain conserved structures associated with the pathogen called as pathogen associated molecular patterns (PAMPs) and activate their first line of defence. PAMPs are usually the structural components on the microbial wall which may be proteins, glycoproteins, lipopolysaccharides or oligosaccharides [1]. Their recognition activates the PAMP-triggered immunity (PTI) in the host which does not allow the pathogen to infect host cells. The pathogens which are able to breach the first line of defense are able to colonize the host and cause disease ([2, 3].Such pathogens secrete an arsenal of effector molecules into the plant cytoplasm or apoplast and help in establishing the virulence of the pathogen. Although the effector proteins are means of the pathogen to modulate host cell physiology to promote susceptibility to pathogens, but some plants have evolved resistance $(\mathrm{R})$ proteins which recognise the effectors and relay the second line of defense, the effector-triggered immunity (ETI), eliciting certain disease responses, that kill the host cell to prevent further spread of the pathogen [4,5] The effectors recognised by the $\mathrm{R}$ proteins are called as Avirulence (Avr) proteins. The pathogens that carry the dominant $A v r$ gene are unable to develop the disease in the host species which carry the dominant cognate $R$ gene because the functional $\mathrm{R}$ and Avr gene products interact directly inside the host leading to a rapid defense responses to check the disease development and thus follow the gene-for-gene hypothesis [6]. Besides classical receptor-ligand model of direct interaction between the $\mathrm{R}$ and the Avr protein, indirect interaction between the two has also been reported which are conciliated by intermediate operative target moecules $[7,8,9]$. Avirulence genes are diverse and belong to several gene families [10].

Gram negative bacterial pathogens have the type III secretion system used to release effectors into host cells $[11,12,13]$. Effectors of many fungal pathogens bear a secretary signal and are delivered directly into the cytoplasm of plant cell [14] or into intercellular spaces such as the apoplast or the xylem sap from where they enter the plant cell [15]. Effectors play a role both in avirulence and virulence. They are the mediators of interaction between the pathogen and host. If the fungal effectors are not recognized by defense system of the plant, they get sorted to certain subcellular compartments of the plant cell where they perform their role to subvert host cellular machinery to establish their virulence [16]. They may move to the nucleus, cytosol, plasma membrane, endoplasmic reticulum, chloroplasts or mitochondria 
$[17,18,19,20]$. Sorting signals direct the intracellular effectors to distinct compartments, they may carry sequence motifs at the $\mathrm{N}$-terminal regions followed after signal peptide such as the oomycete RxLR and Crinkler (CRN) effectors bear the RxLR dEER and the LxFLAK motifs respectively, which direct their movement to the organelle where they act $[21,22]$. The localization of an effector protein in the plant cell is in accordance with its function [23,24]. The effector which localize in the nucleus may affect host machinery by chromatin modeling or by acting as a transcription factor. The bacterial effectors are known to act as transcription factors that directly affect the gene expression. They may change the chromatin configuration by loosening the histone protein or they may even directly target the transcription factors of the host cell [25]. Some of them may be involved in influencing RNA silencing [26] while others may have some enzymatic function such as the Cmul effector from Ustilago maydis acts as chorismate mutase in its host cytoplasm [27]. The subcellular position of effectors in host cells provides knowledge of the function of any Avr proteins and the molecular and cellular basis of their virulence activities. Therefore, effector localization can be used as a selective marker to reveal host cell functioning [28].

Magnaporthe oryzae is a hemibiotrophic heterothallic fungus that causes one of the most dreaded rice diseases called rice blast $[29,30]$. This disease is detrimental to the plant and causes a huge loss of crop yield every year which is estimated to have fed 60 million people of the world for a day [31]. Many rice cultivars which have $\mathrm{R}$ genes are resistant to certain strains of M. oryzae. A broad spectrum resistance gene Pi54 has been cloned and characterised from the rice line Tetep that imparts immunity against various isolates of $M$. oryzae [32,33]. Its cognate effector gene AvrPi54 has also been characterised from M. oryzae. The AvrPi54 interacts directly with the Pi54 protein in Yeast-2-Hybrid analysis [34]. AvrPi54 containing $M$. oryzae strains are known to induce hypersensitive response in the rice containing Pi54 gene $[32,33]$. The immunity responses generated after the recognition of Avr protein in the host cell is an important area to be speculated because the understanding of the exact mechanism of effector triggered immunity (ETI) can allow the use of this component in raising disease resistance and is widely used in breeding programs [1,35]. This component can be engineered to raise transgenics which can assure broad spectrum resistance to the pathogen [36]. Therefore, identification and characterization of effector proteins is a premier step in resistance development in the plant. Therefore, we have attempted to study physiochemical properties of AvrPi54 protein and its subcellular localization to have clue of the nature of its action in the rice cell. The protocol to express and purify the protein has been 
designed. The purified protein can be used to elucidate the structural details and set up in vitro experiments to understand molecular events that occur in its interaction with cognate partner.

\section{Results}

\subsection{In silico determination of physiochemical properties of AvrPi54}

The nascent AvrPi54 protein is composed of 153 amino acid residues. It is rich in Alanine (13.4\%), followed by Valine (11.2\%) and Glycine (9.7\%). It has 4 Cysteine residues (3\%) and 7 negatively charged and 7 positively charged residues (Figure 1A). Two disulfide bonds formation was predicted between cysteine residues: Cys35Cys67 and Cys118Cys153. The DeepLoc-1.0 and WoLF PSORT softwares predicted it to be an extracellular protein of $M$. oryzae. It bears a 19 amino acids signal peptide at the N-terminal. Therefore, after cleaving the signal peptide the mature AvrPi54 402 protein contains 134 amino acids. The size of the His-tagged AvrPi54 ${ }^{402}$ was nearly $15 \mathrm{kDa}$ when expressed in E. coli as seen in SDS PAGE gel. The $6 \mathrm{X}$ His-Tag adds nearly $1 \mathrm{kDa}$ weight to the protein. Therefore, the mature AvrPi54 obtained after processing of signal peptide is approximately a $14 \mathrm{kDa}$ protein; nearly the same as is predicted $(13.8 \mathrm{kDa})$ by in silico approach. The GRAVY index is a small positive number, 0.18 which indicates that the protein is slightly hydrophobic. Hydrophobicity of amino acid residues was in the range of -2 to +2 (Figure 1B). The isoelectric point (pI) of the protein is 6.78 , the $\mathrm{pH}$ when no net charge is present on the protein and it will not migrate even if an external electric field is applied. Aliphatic index, Half-Life, Instability Index indicate that the protein is stable with a thermostable profile under a wide range of temperature variation. All these properties are listed in Table 1.

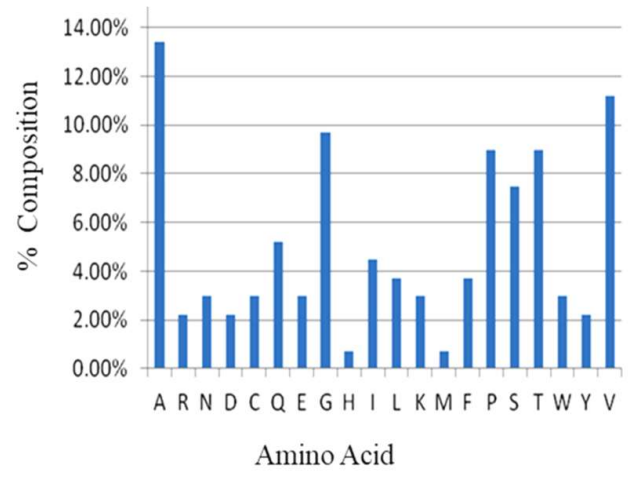

(A)

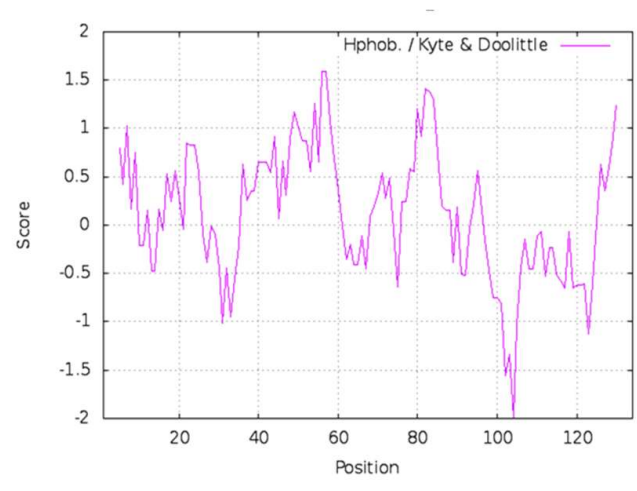

(B)

Figure 1. In silico properties of AvrPi54 protein. (A) percentage of amino acid 
composition of mature AvrPi54 protein. (B) Hydrophobicity curve of amino acids of mature AvrPi54 protein (Kyte and Doolittle).

Table 1. Physiochemical properties of mature AvrPi54 protein

\begin{tabular}{clc}
\hline S. No & \multicolumn{1}{c}{ Physiochemical property } & In silico prediction \\
\hline 1 & Amino acid residues (nascent) & 153 \\
2 & Amino acid residues (mature) & 134 \\
3 & Molecular weight & $13.8 \mathrm{kDa}$ \\
4 & Isoelectric point (pI) & 6.78 \\
5 & $\begin{array}{l}\text { Negatively Charged residues } \\
\text { (Asp + Glu): }\end{array}$ & 7 \\
6 & $\begin{array}{l}\text { Positively Charged residues } \\
\text { (Arg + Lys): }\end{array}$ & 77.91 \\
7 & Alihatic index & 1.693 \\
9 & Extinction coefficient $\left(\mathrm{M}^{-1} \mathrm{~cm}^{-1}\right.$ at 280nm) & 0.18 \\
\hline
\end{tabular}

\subsection{Protein expression and solubility}

The $A v r P i 54^{402}$ insert was amplified and purified. It was cloned at the NdeI and NotI sites of the pET-28a $(+)$ which is a protein expression vector. Double digested by NdeI and NotI restriction enzymes confirms the release of nearly 402 bp insert (Figure 2A). A good amount of recombinant protein expression was obtained upon induction of BL21 (DE3) pLysS cells with $0.5 \mathrm{mM}$ IPTG at $37^{\circ} \mathrm{C}$, carrying pET $28 \mathrm{a}(+) \_$AvrPi5 $54^{402}$. The size of the overexpressed protein was nearly $15 \mathrm{kDa}$ (Figure $2 \mathrm{~B}$ ). It was validated by performing the western blot analysis (Figure 3). The expressed protein was found to be insoluble in all the buffers used for screening except for the urea containing buffers where urea concentration was $3 \mathrm{M}$ or more. Based on this the buffer used to extract the protein from the insoluble fraction of the protein was $50 \mathrm{mM}$ Tris, $\mathrm{pH} 7.5,3 \mathrm{M}$ Urea, $50 \mathrm{mM} \mathrm{NaCl}, 1 \mathrm{mg} / \mathrm{ml}$ lysozyme (3M Urea buffer). 


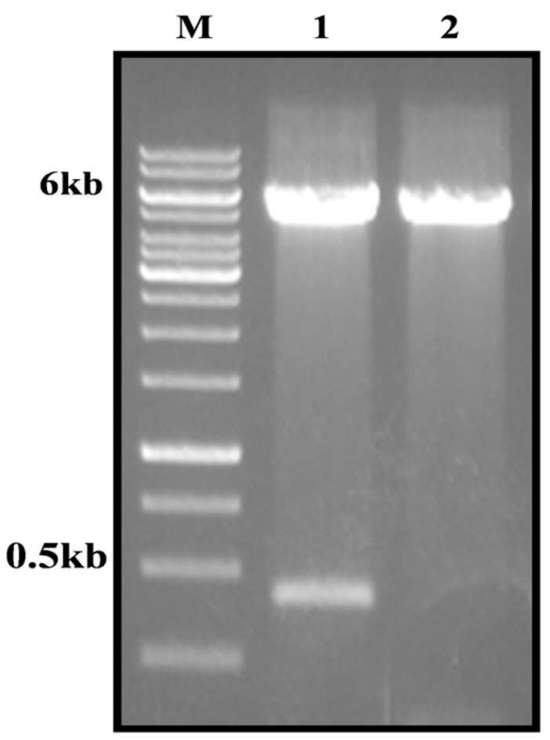

(A)

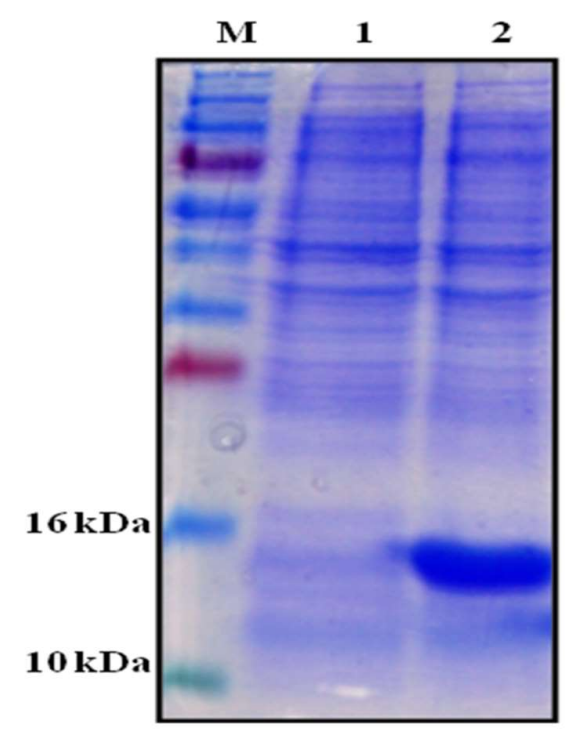

(B)

Figure 2. Cloning and expression of $\mathrm{AvrPi54^{402 }}$ in pET28a(+) vector. (A) Lane 1 shows restriction digestion to confirm the release of cloned insert AvrPi54 402 from recombinant plasmid pET28a $(+)$; Lane 2 shows native pET28a(+) vector digested; with NdeI and NotI enzymes. (B) SDS-PAGE showing in Lane 1 uninduced control protein and in Lane 2 proteins from host transformed with pET-28a $(+) \_$AvrPi54 402 induced with IPTG $(0.5 \mathrm{mM})$ (2). Prominent band of nearly $15 \mathrm{kDa}$ is induced AvrPi54 ${ }^{402}$ protein.

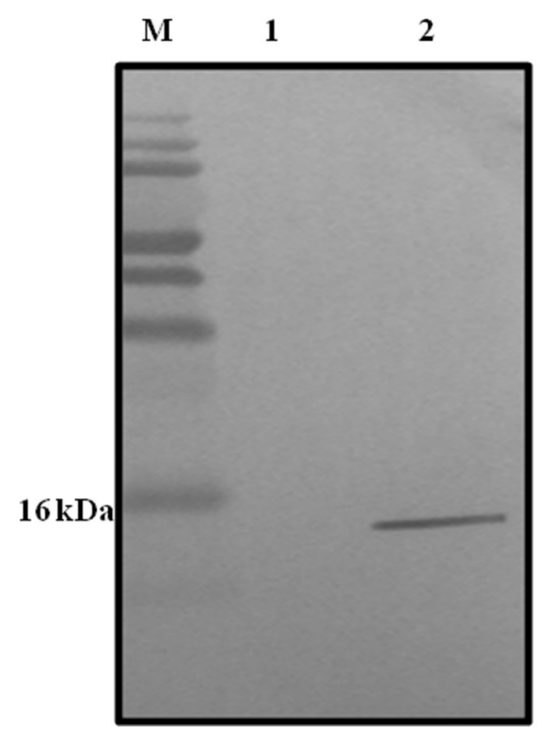

Figure 3. Western blot to confirm the His-tagged AvrPi54 ${ }^{402}$ protein using His-tag antibody. $\mathbf{M}$ is protein ladder; Lane 1 is uninduced control protein sample; Lane 2 is induced 
proteins sample from host transformed with pET-28a $(+) \_$AvrPi54 ${ }^{402}$. The band in Lane 2 corresponds to AvrPi54 ${ }^{402}$ protein obtained after blotting.

\subsection{Purification of AvrPi54 $4^{402}$ protein by using $3 M$ Urea buffer}

Cell fraction containing AvrPi54 ${ }^{402}$ protein was dissolved in $3 \mathrm{M}$ Urea buffer to denature and solubilise the protein. The dialysis of the protein sample mobilised the protein into non urea buffer having $50 \mathrm{mM}$ Tris, $\mathrm{pH} 7.5$ and $50 \mathrm{mM} \mathrm{NaCl}$. And the protein was not seen to precipitate into clumps after refolding. The quality and yield of the purified protein were high (Figure 4).

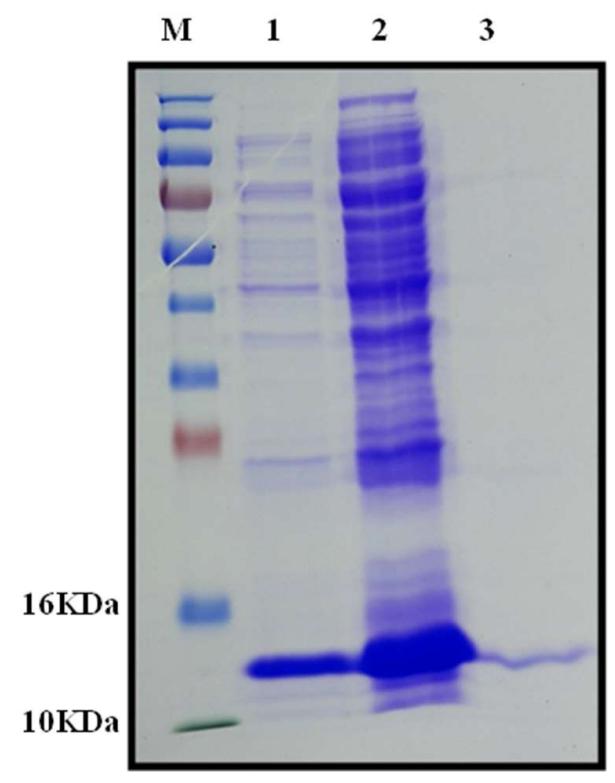

Figure 4. Purification of AvrPi54 $4^{402}$ protein. Lane 1 is flow through containing unbound protein; Lane 2 is induced crude fraction; Lane 3 is purified protein.

\subsection{Avrpi54 localizes to the plasma membrane and nucleus of plant cells}

The pGWB441_AvrPi54 402 construct verification by digesting with with EcoRI and HindIII restriction enzymes released expected products of size 1763, 2462 and $6870 \mathrm{bp}$ and the native vector got digested into fragments of 1336, 1763, 2433 and 6870 with the same enzymes (Figure 5A). The fluorescently tagged protein and control eYFP were successfully delivered into the onion peel epidermal cells using particle bombardment. The localization was then 
examined by confocal microscopy $16 \mathrm{~h}$ post bombardment. The control protein was seen to localize in the membrane, cytoplasm and the nucleus whereas the fluorescence originating in AvrPi54 402 -eYFP fusion protein was mainly found in the plasma membrane and nucleus (Figure 5B). This revealed that AvrPi54 protein sub-localizes to the plasma membrane and nucleus in the plant cell.
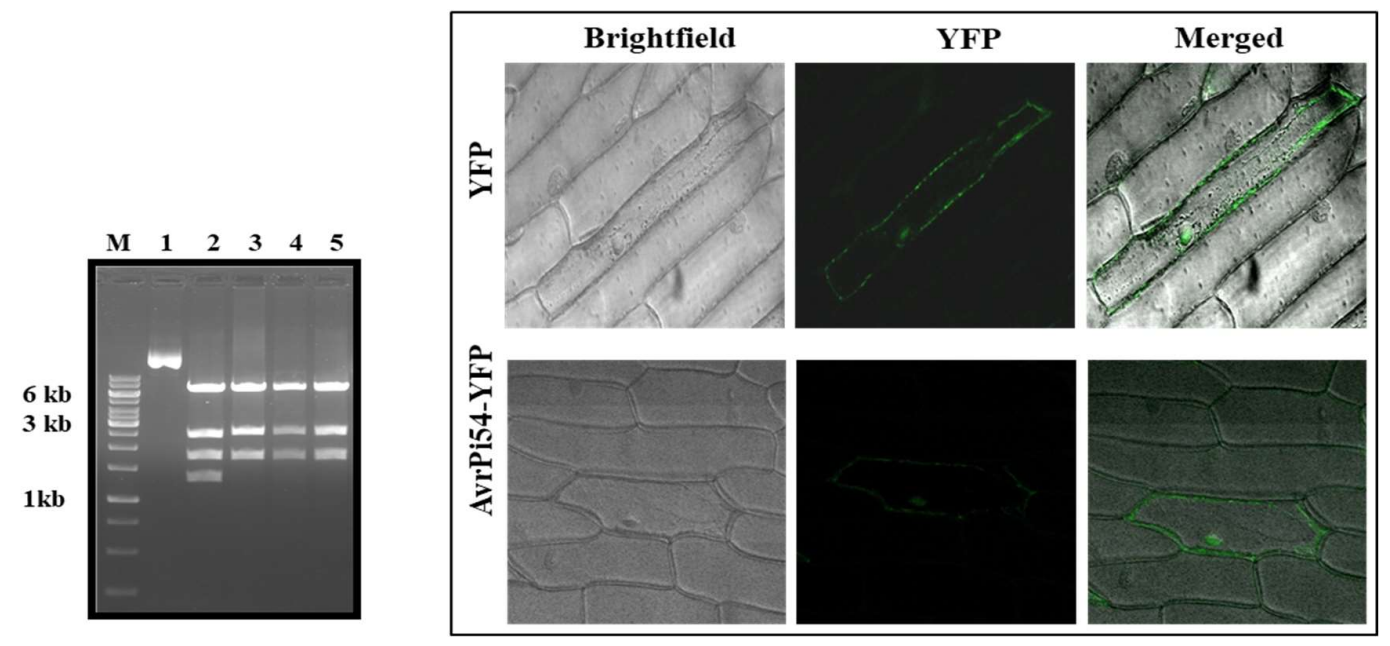

Figure 5. Cloning and confocal microscopy of $\mathrm{AvrPi54} 4^{402}$. (A) Cloning of $\mathrm{AvrPi54^{402 }}$ in pGWB441vector. $\mathrm{M}$ is $1 \mathrm{~kb}$ ladder; Lane 1 is native pGWB441vector; Lane 2 is digested pGWB441 vecror; Lanes 3, 4, 5 shows digested clones. (B) Confocal microscope images for localisation of mature AvrPi54 protein in onion peel epidermal cells.

\section{Discussion}

'Effectors' are the proteins secreted by the pathogens which are the weapons of the pathogen to win over the host innate immune system [37]. Majority of Avr genes cloned from filamentous microorganisms code small secreted proteins without known functions [16]. Homology analysis of AvrPi54 protein did not retrieve significant sequence similarity in the available databases; therefore its function could not be annotated. Generally there is limited homology between most fungal effectors and other proteins present in available databases. One obvious reason is the small number of fungal plant pathogens sequenced, so the databases are still not vast. At the same time, due to the arm race between the host and the pathogen, rapid variation accumulates in the coding regions of the effectors, therefore, majority of the available effectors do not share significant sequence similarity [38], although 
some exceptions have been reported such as the AvrPi-ta [39] and Ace1 [40] effector proteins of M. oryzae and Ecp6 of Cladosporium fulvum [41].

The primary sequence of the protein determines its physiochemical properties which in turn sheds light on its biological function [42]. Our studies have analysed various properties of the AvrPi54 protein using in silico and in vitro methods. Like majority effector proteins, it is also small in size. Having length of 134 amino acid residues, the molecular weight of the processed AvrPi54 protein is nearly $13.8 \mathrm{kDa}$. Traditionally, effectors have been known to contain less than 150 amino acids [16]. However, the number of the amino acid residues in the so far characterized Avr proteins from M. oryzae range between 63 and 314 except for Ace1 which is 4035 amino acids long [43,44]. AvrPi54 shows high amount of small amino acids like Alanine, Valine and Glycine which is very much a characteristic of many proteins that are secreted, a feature with effector proteins $[42,45]$. Due to very small size and least steric hindrances, glycine enrichment has been commonly found in fungal effectors such as Pwl series of Avr proteins and several pathogenicity related small secreted proteins of $M$. oryzae $[42,46,47]$. Majority effectors are also found to have at least four or more cysteine residues [48]; however, many effector proteins do not conform to this criterion. Usually the number of cysteines is even and these are involved in intramolecular bond formation [43]. AvrPi54 protein contains four cysteine residues at position 35, 67, 118 and 153 in the full naïve protein with probable bonds between Cys35Cys67 and Cys118Cys153. The disulfide bonds between cysteine residues provide stability and are crucial for their activity of most of the effector proteins for causing induction of defense responses, however not all cysteine residues are involved in disulfide bridges [43]. NMR spectroscopy has revealed that AvrPiz-t contains a $\beta$-sandwich comprised of six strands and a Cys62Cys 75 disulfide linkage and two other cysteine residues, Cys 23 and Cys 70 are present on the $\beta 1$ sheet and loop 4, respectively [49]. It has also been reported earlier that the avirulence of AvrPiz-t is quashed if mutation occurs at a single residue out of the four cysteine residues [50]. Another feature of AvrPi54 protein is hydrophobicity which is found in some proteins like hydrophobins, found in fungi, which function in pathogenicity [51].

The major repertoire of M. oryzae Avr genes are secretary in nature. Eleven Avr genes are cloned from M. oryzae, namely: Pwl1, Pwl2 [46,47], AvrPi-ta [39], Avr1Co39 [52], Ace1 [40], AvrPiz-t [53], Avr-Pia [54], Avr-Pii [54], Avr-Pik/km/kp [54], AvrPi9 [55], AvrPib [56]. Ace1 is the only characterised effector which is nonsecretory [40]. The naïve AvrPi54 protein has 153 amino acids and the first 19 amino acids at the $\mathrm{N}$-terminal end constitute the signal 
peptide and are responsible for secretion of AvrPi54 protein from the cell. The N-terminal secretion signal is critical for the Avr function as it is needed for secretion of the protein from the pathogen into the host cell [53]. The virulent isolate of M. oryzae when transformed with a construct containing AvrPiz- $t$ without predicted signal peptide could not impart avirulence to it against Piz-t protein, confirming the essentiality of secretion of effectors for their function [53]. It has also been seen that Ps 87 protein of P. striiformis is a secreted protein having N-terminal signal peptide and is an effector protein whereas its homolog Mg87 protein in M. oryzae is non-secretory that does not have the signal peptide for its secretion and hence it is a nonpathogenic protein [57]. The presence of the signal peptide is probably responsible for the recruitment of Ps87 protein as an effector, unlike its homolog which is retained in the pathogen cell [57]. Yeast-2-Hybrid analysis and in silico docking analysis have shown stronger interaction potential between the mature AvrPi54 ${ }^{402}$ protein and Pi54 protein compared to interaction between its full length AvrPi54 protein and Pi54 protein [34]. This indicates that AvrPi54 protein is processed for cleavage of the signal peptide probably during its secretion or later in the host cell. Many effectors are processed at the $\mathrm{N}$ - and $\mathrm{C}$ terminal regions by either fungal and/or plant proteases [9]. The signal peptides of the effector protein such as the Avr and Ecp effectors of Cladosporium fulvum and the Nip effectors of Rhynchosporium secalis are cleaved off and the mature proteins are released into the host $[9,58]$. The Six effectors characterised from Fusarium oxysporum are also processed [59]. In our study, the mature fungal AvrPi54 protein was expressed in E. coli cells under the control of $\mathrm{T} 7$ promoter. The protein aggregates into the inclusion bodies when expressed which may be attributed to the native intramolecular sulfide linkages that occur in the protein. The urea-based buffers denature the protein and solubilise the inclusion bodies [60]. Protein can be renatured by slow removal of urea from inclusion and purified using affinity chromatography. The purification in denaturing conditions using Urea added buffer yielded a higher quantity of the purified protein.

The fungal effectors do not have consensus signature that which would otherwise have clues for their function in translocation and entry into the plant cell. Sub cellular localization analysis provides an insight into the in vivo behavior of proteins. This subcellular localization study was done to know the localization of the AvrPi5 protein in cellular compartments. AvrPi54 protein tagged to YFP when moved to onion peel epidermal cell is seen to be located in the cytoplasmic face of plasma membrane and the nucleus of the onion cell. This implies that when AvrPi54 protein is released by M. oryzae into rice cells after invading the plant, it 
possibly gets located in the inner plasma membrane facing the cytoplasm and the nucleus. The UfRTP1 protein, a cysteine protease inhibitor from Uromyces fabae that has a nuclear localization signal (NLS), is transferred into the nucleus and cytoplasm of infected plant cells [61]. Nuclear targeting of effectors is the virulence strategy documented in many bacteria, plant pathogenic viruses and fungi [62]. In the nucleus of the plant cell, effectors may be able to subvert the host transcription machinery in favor of the pathogen. Some bacterial effectors like Transcription activator-like (TAL) effectors which function like transcriptional activators in the nucleus of host cell, binds directly to DNA to regulate expression of genes involved the disease development $[63,64]$. The effectors on reaching the nucleus may conjugate with other molecules to modify the chromatin configuration by influencing the interaction between histone proteins and DNA. This allows access to new regions on DNA and their expression which may give advantage to the pathogen [65]. The effectors are also known to alter the function of host transcription factors to manipulate their function and promote virulence. In Arabidopsis, transcription factor MYB30, which regulates the defense related genes, is the target of effector XopD. [66].

The functional myristoylation motif presence in some effectors directs them to the cytoplasmic surfaces of the membranes. The AvrPto protein, of Pseudomonas syringae, pathogen of tomato and tobacco, find its way to the plasma membrane of the host [67]. Mutation in myristoylation motif in AvrPto abolishes its function of avirulence [68]. Rpm1, the $\mathrm{R}$ gene of Arabidopsis is a peripheral plasma membrane and its cognate AvrRpm1 in $P$. syringae, bears the myristoylation motif and is possibly targeted to the inner plasma membrane surface [69]. The Avr and Six effectors of Cladosporium fulvum (Cf) are secreted into the apoplastic region of the host from where Avr2 (Six3) and Six5 are translocated into the cytoplasm. Most of the fungal effector proteins are found to localize in apoplast and cytoplasm of the plant cell. The Six effectors of the fungus Fusarium oxysporium f. sp. lycopersici are translocated to the xylem tissue [3]. Some of the cytoplasmic Avrs of $M$. oryzae probably have enzymatic activity [70].

Many NBS-LRR proteins are transmembrane proteins [71], while others may be located peripherally with membranes [69]. Many Avr proteins also are localized to plant membranes [67,72]. NBS- LRR domain containing Pi54 resistant protein has been predicted to be anchored in the plasma membrane [32]. The distribution of AvrPi54 protein in the plant cell membrane matches the putative location of its cognate Pi54 protein. In the nucleus, AvrPi54 may conduct its primary function to suppress the immune system of the host by either acting 
as a transcription factor or a chromatin remodeling factor to alter the gene response in host where the Pi54 protein is not functional. Thus, AvrPi54 probably exert its virulence strategy to affect the protein turnover in the host and modify the pathogenesis-related processes. But in resistant cells the AvrPi54 is recognized at the plasma membrane by the LRR domain of Pi54 protein which may be membrane localized, as are many $R$ genes and this interaction thus leads to the downstream signal cascade. This cascade triggers the expression of defense response genes and therefore the hypersensitive response is generated which leads to cell death and isolation of the pathogen from other living cells.

\section{Materials and methods}

\subsection{In silico determination of physiochemical properties of AvrPi54}

The nucleotide sequence of AvrPi54 was downloaded from NCBI (Accession no. HF545677) and translated into amino acid sequence using the ExPASy-Translate tool (https://web.expasy.org/translate/). To predict the subcellular localization of the protein in the fungal cell, two prediction softwares, DeepLoc-1.0 (http://www.cbs.dtu.dk/services/DeepLoc/) [73] and WoLF PSORT (https://wolfpsort.hgc.jp/aboutWoLF_PSORT.html.en) [74 were used. The SignalP4.1 server (http:/www.cbs.dtu.dk/services/SignalP/) ) and the TargetP1.1 (www.cbs.dtu.dk/services/TargetP/) [75] were used to get the location and size of the signal peptide. The homology search of AvrPi54 was performed using Hhpred software (https://omictools.com/hhpred-tool). The physiochemical properties of the mature AvrPi54 protein (AvrPi54 ${ }^{402}$ ), without signal peptide sequence, were determined using ProtParam module of ExPASy (http://web.expasy.org/protparam/) [76]. Molecular mass was estimated using the Isotopident tool of the ExPASy (http://education.expasy.org/student_projects/isotopident/htdocs/). The ProtScale tool in ExPASy (http://web. expasy.org/protscale/), was used to analyse the hydropathicity curve of constituent amino acids. DiANNA 1.1 web server was used to predict the possible disulfide bonds in the protein [77].

\subsection{Cloning and Expression of AvrPi54 402}

Primers were designed from the 402 bp insert of AvrPi54_ORF after removing the signal peptide sequence to amplify the AvrPi5 $4^{402}$ fragment from DNA of M. oryzae RML-29 strain. The forward primer contained a restriction site for $N d e$ I enzyme while the reverse primer was designed without the stop codon to allow the addition of the $6 \mathrm{X}$-His tag at the C-terminal of 
the protein from pET-28a(+) vector and the restriction site for the NotI enzyme. AvrPi54 402 fragment was then cloned into $\mathrm{pET}-28 \mathrm{a}(+)$ vector and transformed into the E.coli BL21(DE3)pLysS expression system. Expression was induced by addition of $0.5 \mathrm{mM}$ isopropylthio- $\beta$-D-galactopyranoside (IPTG) to the transformed bacterial culture with incubation for $4-5 \mathrm{~h}$ at $37^{\circ} \mathrm{C}$. The crude protein sample was mixed with $5 \mathrm{X}$ sample loading buffer (Thermo Scientific, USA) and denatured at $100^{\circ} \mathrm{C}$ for $5 \mathrm{~min}$ and was analysed on $12 \%$ SDS-PAGE.

\subsection{Western blotting}

For western blot analysis, the unstained SDS-PAGE proteins were transferred onto a PVDF (GE Healthcare) membrane in the Trans-Blot Semi-Dry cell (Bio-Rad) using an electroblotting buffer (25 mM Tris- $\mathrm{HCl}, 190 \mathrm{mM}$ glycine, $20 \%$ methanol) at $15 \mathrm{~V}$ for $1 \mathrm{~h}$ at room temperature. The membrane was blocked with $5 \%$ skim milk in TBST buffer $(20 \mathrm{mM}$ Tris, $\mathrm{pH} 7.5,150 \mathrm{mM} \mathrm{NaCl}, 0.1 \%$ Tween 20 ). The detection of recombinant protein was performed with Anti-His(C-term)-HRP antibody procured from Invitrogen (life technologies) following manufacturers protocol. The blot was developed using the 3,3',5,5'Tetramethylbenzidine (TMB) (Thermo Scientific) a chromogenic substrate.

\subsection{Determination of protein solubility}

The solubility of the expressed protein was determined by dissolving the pellet in the solubility screens mentioned by the European Molecular Biology Laboratory (EMBL) (https://www.embl.de/pepcore/pepcore_services/protein_purification/extraction_clarification/ solubility_studies/index.html). After sonication, the soluble fraction was separated by centrifugation of the sample at $10000 \mathrm{~g}$ at $4^{\circ} \mathrm{C}$ for $30 \mathrm{~min}$ and analysed on $12 \%$ SDS-PAGE.

\subsection{Purification of AvrPi5 $4^{402}$ protein by using $3 M$ Urea buffer}

For purification, $100 \mathrm{ml}$ culture of E.coli strain BL21(DE3)pLysS transformed with pET$28 \mathrm{a}(+) \_$AvrPi5 $54^{402}$ was used. All steps were performed at $4^{\circ} \mathrm{C}$. The cells were harvested by centrifugation at $4000 \mathrm{~g}$ for $20 \mathrm{~min}$. $20 \mathrm{ml}$ of $3 \mathrm{M}$ Urea buffer was used to resuspend the pellet. The cells were sonicated. After centrifugation at $10000 \mathrm{~g}$ for $30 \mathrm{~min}$, the supernatant was dialyzed against buffers having decreasing gradients of urea, $2 \mathrm{M}$ and then $1 \mathrm{M}$ urea buffer and finally to the buffer containing $50 \mathrm{mM}$ Tris, $\mathrm{pH} 7.5$ and $50 \mathrm{mM} \mathrm{NaCl}$. The supernatant of the cell lysate was passed through Ni-NTA Agarose packed in a column. Buffer containing the recombint protein was slowly passed through the column to allow effective binding to the column resin. Finally, the bound protein was eluted with buffers 
having increasing imidazole concentrations $(100 \mathrm{mM}, 150 \mathrm{mM}$ and $200 \mathrm{mM}$ in lysis buffer ( $\mathrm{pH}$ 8.0). The eluted fractions were concentrated using Protein Concentrator PES (Thermo Scientific). $5 \mu \mathrm{l}$ of the obtained fractions of the flow-throughs and elutes were mixed with $5 \mathrm{X}$ sample loading buffer and were denatured at $95{ }^{\circ} \mathrm{C}$ for $5 \mathrm{~min}$. These fractions were loaded to the $12 \%$ SDS-PAGE.

\subsection{Localisation study}

Recombinant plasmid for localization pGWB441_AvrPi54 $4^{402}$ was prepared by using gateway

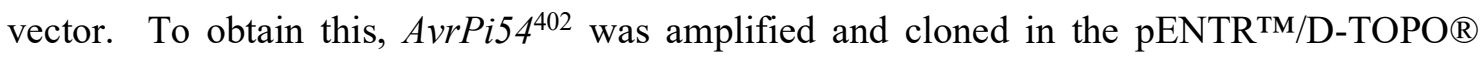
vector according to the protocol recommended in manual for $\mathrm{pENTR}^{\mathrm{TM}}$ Directional TOPO ${ }^{\circledR}$ Cloning Kit (invitrogen). It was shuttled into pGWB441 destination vector containing the yellow fragment protein (YFP). pGWB441 was a gift from Tsuyoshi Nakagawa (Addgene plasmid \#74827; http://n2t.net/addgene:74827; RRID:Addgene_74827) [78]. After confirming translational fusion the construct was transiently expressed in onion epidermal cells by particle bombardment with a PDS-1000/He system (www.bio-rad.com) and transformants were analyzed by confocal microscopy (LSM 880, Carl Zeiss Microscopy GmbH 1997-2015).

\section{Conclusion}

AvrPi54 gene of M. oryzae, encodes a nascent secretory protein of 153 amino acids which is processed to 134 amino acids long mature protein. The purified protein is essential to be used for in vitro assays to study its interaction with cognate protein and for the biophysical structure determination. Lack of homology does not allow the prediction of its putative role, therefore suitable strategy has to be designed to characterise it functionally to have a better understanding of its function and role in pathogenicity.

Author Contributions: Authors' contributions: B.K.S. and T.R.S. conceived the research plans; B.K.S. did the experiments; B.K.S. and K.A. analyzed the data; B.K.S. drafted the first manuscript; T.R.S. and R.K.A supervised the overall project and finalized the writing.

Acknowledgments: TRS is thankful to the Department of Biotechnology, Govt. of India for financial help and Department of Science and Technology, Govt of India for JC Bose National Fellowship. BKS is thankful to CSIR, New Delhi, India for providing financial assistance in the form of Fellowship.

Conflict of interest: The authors declare no conflict of interest 


\section{References}

1. Zipfel, C.; Robatzek, S. Pathogen-associated molecular pattern-triggered immunity: veni, vidi...?. Plant Physiol. 2010, 154, 551-554.

2. Göhre, V.; Robatzek, S. Breaking the barriers: microbial effector molecules subvert plant immunity. Annu. Rev. Phytopathol. 2008, 46, 189-215.

3. De Wit, P.J.; Joosten, M.H.; Thomma, B.H.; Stergiopoulos, I. Gene for gene models and beyond: the Cladosporium fulvum-Tomato pathosystem. In Plant relationships; Springer, Berlin, Heidelberg 2009, 135-156.

4. Jones, J.D.; Dangl, J.L. The plant immune system. Nature 2006, 444, 323.

5. Spoel, S.H.; Dong, X. How do plants achieve immunity? Defence without specialized immune cells. Nat. Rev. immunol. 2012, 12, 89.

6. Flor, H.H. Host-parasite interactions in flax rust-its genetics and other implications. Phytopathol. 1955, 45, 680-685.

7. Zhou, J.; Loh, Y.T.; Bressan, R.A.; Martin, G.B. The tomato gene Ptil encodes a serine/threonine kinase that is phosphorylated by Pto and is involved in the hypersensitive response. Cell 1995, 83, 925-935.

8. Van Der Biezen, E.A.; Jones, J.D. Plant disease-resistance proteins and the gene-forgene concept. Trends biochem. sci. 1998, 23, 454-456.

9. Zipfel, C.; Rathjen, J.P. Plant immunity: AvrPto targets the frontline. Curr. Biol. 2008, 18, R218-R220.

10. White, F.F.; Yang, B.; Johnson, L.B. Prospects for understanding avirulence gene function. Curr. Opin. Plant Biol. 2000, 3, 291-298.

11. Galán, J.E.; Wolf-Watz, H. Protein delivery into eukaryotic cells by type III secretion machines. Nature 2006, 444, 567-73.

12. Block, A.; Li, G.; Fu, Z.Q.; Alfano, J.R. Phytopathogen type III effector weaponry and their plant targets. Curr. Opin. Plant Biol. 2008, 11, 396-403.

13. McCann, H.C.; Guttman, D.S. Evolution of the type III secretion system and its effectors in plant-microbe interactions. New Phytol. 2008, 177, 33-47.

14. Zhang, S.; Xu, J.R. Effectors and effector delivery in Magnaporthe oryzae. PLoS pathog. 2014, 10, 1003826.

15. Torto, T.A.; Li, S.; Styer, A.; Huitema, E.; Testa, A.; Gow, N.A.; Van West, P.; Kamoun, S. EST mining and functional expression assays identify extracellular 
effector proteins from the plant pathogen Phytophthora. Genome res. 2003, 13, pp.1675-1685.

16. Kamoun, S. A catalogue of the effector secretome of plant pathogenic oomycetes. Annu. Rev. Phytopathol. 2006, 44, 41-60.

17. Jelenska, J.; Yao, N.; Vinatzer, B.A.; Wright, C.M.; Brodsky, J.L.; Greenberg, J.T. AJ domain virulence effector of Pseudomonas syringae remodels host chloroplasts and suppresses defenses. Curr. Biol. 2007, 17, 499-508.

18. Caillaud, M.C.; Piquerez, S.J.; Fabro, G.; Steinbrenner, J.; Ishaque, N.; Beynon, J.; Jones, J.D. Subcellular localization of the Hpa RxLR effector repertoire identifies a tonoplast-associated protein HaRxL17 that confers enhanced plant susceptibility. Plant J. 2012, 69, 252-265.

19. Hicks, S.W.; Galán, J.E. Exploitation of eukaryotic subcellular targeting mechanisms by bacterial effectors. Nat. Rev. Microbiol. 2013, 11, 316.

20. Li, G.; Froehlich, J.E.; Elowsky, C.; Msanne, J.; Ostosh, A.C.; Zhang, C.; Awada, T.; Alfano, J.R. Distinct Pseudomonas type-III effectors use a cleavable transit peptide to target chloroplasts. Plant J. 2014, 77, 310-321.

21. Petre, B.; Kamoun, S. How do filamentous pathogens deliver effector proteins into plant cells? PLoS Biol. 2014, 12, e1001801.

22. Gascuel, Q.; Buendia, L.; Pecrix, Y.; Blanchet, N.; Muños, S.; Vear, F.; Godiard, L. RXLR and CRN effectors from the sunflower downy mildew pathogen Plasmopara halstedii induce hypersensitive-like responses in resistant sunflower lines. Front. Plant Sci. 2016, 7, 1887.

23. Alfano, J.R. Roadmap for future research on plant pathogen effectors. Mol. plant pathol. 2009, 10, 805-813.

24. Schornack, S.; van Damme, M.; Bozkurt, T.O.; Cano, L.M.; Smoker, M.; Thines, M.; Gaulin, E.; Kamoun, S.; Huitema, E. Ancient class of translocated oomycete effectors targets the host nucleus. Proc. Natl. Acad. Sci. U.S.A. 2010, 107, 17421-17426.

25. Canonne, J.; Rivas, S. Bacterial effectors target the plant cell nucleus to subvert host transcription. Plant Signal Behav. 2012, 7, 217-221.

26. Qiao, Y.; Shi, J.; Zhai, Y.; Hou, Y.; Ma, W. Phytophthora effector targets a novel component of small RNA pathway in plants to promote infection. Proc. Natl. Acad. Sci. U.S.A. 2015, 112, 5850-5855. 
27. Djamei, A.; Schipper, K.; Rabe, F.; Ghosh, A.; Vincon, V.; Kahnt, J.; Osorio, S.; Tohge, T.; Fernie, A.R.; Feussner, I.; et al. Metabolic priming by a secreted fungal effector. Nature 2011, 478, 395.

28. Varden, F.A.; De la Concepcion, J.C.; Maidment, J.H.; Banfield, M.J. Taking the stage: effectors in the spotlight. Curr. Opin. Plant Biol. 2017, 38, 25-33.

29. Fisher, M.C.; Henk, D.A.; Briggs, C.J.; Brownstein, J.S.; Madoff, L.C.; McCraw, S.L.; Gurr, S.J. Emerging fungal threats to animal, plant and ecosystem health. Nature 2012, 484, 186.

30. Wilson, R.A.; Talbot, N.J. Under pressure: investigating the biology of plant infection by Magnaporthe oryzae. Nat. Rev. Microbiol. 2009, 7, 185.

31. Talbot, N.J. On the trail of a cereal killer: exploring the biology of Magnaporthe grisea. Annu. Rev. Microbiol. 2003, 57,177-202.

32. Sharma, T.R.; Madhav, M.S.; Singh, B.K.; Shanker, P.; Jana, T.K.; Dalal, V.; Pandit, A.; Singh, A.; Gaikwad, K.; Upreti, H.C.; et al. High-resolution mapping, cloning and molecular characterization of the Pi-k h gene of rice, which confers resistance to Magnaporthe grisea. Mol. Genet. Genomics 2005, 274, 569-578.

33. Rai, A.K.; Kumar, S.P.; Gupta, S.K.; Gautam, N.; Singh, N.K.; Sharma, T.R. Functional complementation of rice blast resistance gene Pi-k h (Pi54) conferring resistance to diverse strains of Magnaporthe oryzae. J. Plant. Biochem. Biot. 2011, 20, $55-65$.

34. Ray, S.; Singh, P.K.; Gupta, D.K.; Mahato, A.K.; Sarkar, C.; Rathour, R.; Singh, N.K.; Sharma, T.R. Analysis of Magnaporthe oryzae genome reveals a fungal effector, which is able to induce resistance response in transgenic rice line containing resistance gene, Pi54. Front. plant sci. 2016, 7, 1140.

35. Sharma, T.R.; Rai, A.K.; Gupta, S.K.; Vijayan, J.; Devanna, B.N.; Ray, S. Rice blast management through host-plant resistance: retrospect and prospects. Agric. Res 2012, 1, 37-52.

36. de Wit, P.J. Molecular characterization of gene-for-gene systems in plant-fungus interactions and the application of avirulence genes in control of plant pathogens. Annu. Rev. phytopathol. 1992, 30, 391-418.

37. Dangl, J.L.; McDowell, J.M. Two modes of pathogen recognition by plants Proc. Natl. Acad. Sci. U.S.A. 2006, 103, 8575-8576. 
38. Sperschneider, J.; Dodds, P.N.; Gardiner, D.M.; Manners, J.M.; Singh, K.B.; Taylor, J.M. Advances and challenges in computational prediction of effectors from plant pathogenic fungi. PLoS pathog. 2015, 11, 1004806.

39. Orbach, M.J.; Farrall, L.; Sweigard, J.A.; Chumley, F.G.; Valent, B. A telomeric avirulence gene determines efficacy for the rice blast resistance gene Pi-ta. Plant Cell 2000, 12, 2019-2032.

40. Böhnert, H.U.; Fudal, I.; Dioh, W.; Tharreau, D.; Notteghem, J.L.; Lebrun, M.H. A putative polyketide synthase/peptide synthetase from Magnaporthe grisea signals pathogen attack to resistant rice. Plant Cell 2004, 16, 2499-2513.

41. Bolton, M.D.; Van Esse, H.P.; Vossen, J.H.; De Jonge, R.; Stergiopoulos, I.; Stulemeijer, I.J.; Van Den Berg, G.C.; Borrás-Hidalgo, O.; Dekker, H.L.; De Koster, C.G.; De Wit, P.J. The novel Cladosporium fulvum lysin motif effector Ecp6 is a virulence factor with orthologues in other fungal species. Mol. microbiol. 2008, 69, 119-136.

42. Ray, S.; Vijayan, J.; Singh, P.K.; Sharma, T.R. Identification of pathogenicity related small secreted proteins from Magnaporthe oryzae by comparison of predicted proteome with nonpathogenic relative, Neurospora crassa. ORYZA-An International J. Rice 2014, 51, 183-194.

43. Stergiopoulos, I.; de Wit, P.J. Fungal effector proteins. Annu. Rev. phytopathol. 2009, 47, 233-263.

44. Selin, C.; de Kievit, T.R.; Belmonte, M.F.; Fernando, W.G. Elucidating the role of effectors in plant-fungal interactions: progress and challenges. Front. Microbiol. 2016, 7, 600 .

45. Sperschneider, J.; Gardiner, D.M.; Taylor, J.M.; Hane, J.K.; Singh, K.B.; Manners, J.M. A comparative hidden Markov model analysis pipeline identifies proteins characteristic of cereal-infecting fungi. BMC genomics 2013, 14, 807.

46. Sweigard, J.A.; Carroll, A.M.; Kang, S.; Farrall, L.; Chumley, F.G.; Valent, B. Identification, cloning, and characterization of PWL2, a gene for host species specificity in the rice blast fungus. Plant cell 1995, 7, 1221-1233.

47. Kang, S.; Sweigard, J.A.; Valent, B. The PWL host specificity gene family in the blast fungus Magnaporthe grisea. Mol. Plant Microbe Interact. 1995, 8, 939-948. 
48. Ellis, J.G.; Rafiqi, M.; Gan, P.; Chakrabarti, A; Dodds, P.N. Recent progress in discovery and functional analysis of effector proteins of fungal and oomycete plant pathogens. Curr. Opin. Plant Biol. 2009, 12, 399-405.

49. Zhang, Z.M.; Zhang, X.; Zhou, Z.R.; Hu, H.Y.; Liu, M.; Zhou, B.; Zhou, J. Solution structure of the Magnaporthe oryzae avirulence protein AvrPiz-t. J. biomol. NMR 2013, 55, 219-223.

50. Li, P.; Bai, B.; Zhang, H.Y.; Zhou, H.; Zhou, B. Genomic organization and sequence dynamics of the AvrPiz-t locus in Magnaporthe oryzae.J. Zhejiang Univ-Sc. B 2012, 13, 452-464.

51. Bayry, J.; Aimanianda, V.; Guijarro, J.I.; Sunde, M.; Latge, J.P. Hydrophobinsunique fungal proteins. PLoS pathog. 2012, 8, 1002700.

52. Farman, M.L.; Leong, S.A. Chromosome walking to the AVR1-CO39 avirulence gene of Magnaporthe grisea: discrepancy between the physical and genetic maps. Genetics 1998, 150, 1049-1058.

53. Li, W.; Wang, B.; Wu, J.; Lu, G.; Hu, Y.; Zhang, X.; Zhang, Z.; Zhao, Q.; Feng, Q.; Zhang, H.; et al. The Magnaporthe oryzae avirulence gene AvrPiz-t encodes a predicted secreted protein that triggers the immunity in rice mediated by the blast resistance gene Piz-t. Mol. Plant Microbe Interact. 2009,22, 411-420.

54. Yoshida, K.; Saitoh, H.; Fujisawa, S.; Kanzaki, H.; Matsumura, H.; Yoshida, K.; Tosa, Y.; Chuma, I.; Takano, Y.; Win, J.; Kamoun, S. Association genetics reveals three novel avirulence genes from the rice blast fungal pathogen Magnaporthe oryzae. Plant Cell 2009, 21, 1573-1591.

55. Wu, J.; Kou, Y.; Bao, J.; Li, Y.; Tang, M.; Zhu, X.; Ponaya, A.; Xiao, G., Li, J., Li, C.; et al. Comparative genomics identifies the Magnaporthe oryzae avirulence effector AvrPi9 that triggers Pi9-mediated blast resistance in rice. New Phytologist 2015, 206, $1463-1475$.

56. Zhang, S.; Wang, L.; Wu, W.; He, L.; Yang, X.; Pan, Q. Function and evolution of Magnaporthe oryzae avirulence gene AvrPib responding to the rice blast resistance gene Pib. Sci. rep. 2015, 5, 11642.

57. Gu, B.; Kale, S.D.; Wang, Q.; Wang, D., Pan, Q.; Cao, H.; Meng, Y., Kang, Z.; Tyler, B.M.; Shan, W. Rust secreted protein Ps87 is conserved in diverse fungal pathogens and contains a RXLR-like motif sufficient for translocation into plant cells. PLoS One 2011, 6, 27217. 
58. Wevelsiep, L.; Kogel, K.H.; Knogge, W. Purification and characterization of peptides from Rhynchosporium secalis inducing necrosis in barley. Physiol. Mol. plant pathol.1991, 39, 71-482.

59. Houterman, P.M.; Speijer, D.; Dekker, H.L.; de Koster, C.G.; Cornelissen, B.J.; Rep, M. The mixed xylem sap proteome of Fusarium oxysporum-infected tomato plants. Mol. plant pathol. 2007, 8, 215-221.

60. Singh, S.M.; Panda, A.K. Solubilization and refolding of bacterial inclusion body proteins. J. biosci. bioeng. 2005, 99, 303-310.

61. Pretsch, K.; Kemen, A.; Kemen, E.; Geiger, M.; Mendgen, K.; Voegele, R. The rust transferred proteins - a new family of effector proteins exhibiting protease inhibitor function. Mol. plant pathol. 2013, 14, 96-107.

62. Yang, B.; Zhu, W.; Johnson, L.B.; White, F.F. The virulence factor AvrXa7 of Xanthomonas oryzae pv. oryzae is a type III secretion pathway-dependent nuclearlocalized double-stranded DNA-binding protein. Proc. Natl. Acad. Sci. U.S.A. 2000, 97, 9807-9812.

63. Boch, J.; Scholze, H.; Schornack, S.; Landgraf, A.; Hahn, S.; Kay, S.; Lahaye, T.; Nickstadt, A.; Bonas, U. Breaking the code of DNA binding specificity of TAL-type III effectors. Science 2009, 326, 1509-1512.

64. Moscou, M.J.; Bogdanove, A.J. A simple cipher governs DNA recognition by TAL effectors. Science 2009, 326, 1501-1501.

65. Ma, K.W.; Flores, C.; Ma, W. Chromatin configuration as a battlefield in plantbacteria interactions. Plant Physiol. 2011, 157, 535-543.

66. Canonne, J.; Marino, D.; Jauneau, A.; Pouzet, C.; Brière, C.; Roby, D; Rivas, S. The Xanthomonas type III effector XopD targets the Arabidopsis transcription factor MYB30 to suppress plant defense. Plant Cell 2011, 23, 3498-3511.

67. Shan, L.; Thara, V.K.; Martin, G.B.; Zhou, J.M.; Tang, X. The Pseudomonas AvrPto protein is differentially recognized by tomato and tobacco and is localized to the plant plasma membrane. Plant Cell 2000, 12, 2323-2337.

68. Shan, L.; He, P.; Zhou, J.M.; Tang, X. A cluster of mutations disrupt the avirulence but not the virulence function of AvrPto. Mol plant-microbe interact. 2000, 13, 592598.

69. Boyes, D.C.; Nam, J.; Dangl, J.L. The Arabidopsis thaliana RPM1 disease resistance gene product is a peripheral plasma membrane protein that is degraded coincident with 
the hypersensitive response. Proceedings of the Natl. Acad. Sci. 1998, 95, 1584915854.

70. Khang, C.H.; Park, S.Y.; Lee, Y.H.; Valent, B; Kang, S. Genome organization and evolution of the AVR-Pita avirulence gene family in the Magnaporthe grisea species complex. Mol. Plant Microbe Interact., 2008, 21, 658-670.

71. Bogdanove, A.J. Protein-protein interactions in pathogen recognition by plants. Plant Mol. Biol. 2002, 50, 981-989.

72. Nimchuk, Z.; Marois, E.; Kjemtrup, S.; Leister, R.T.; Katagiri, F.; Dangl, J.L. Eukaryotic fatty acylation drives plasma membrane targeting and enhances function of several type III effector proteins from Pseudomonas syringae. Cell 2000, 101, 353363.

73. Almagro Armenteros, J.J.; Sønderby, C.K.; Sønderby, S.K.; Nielsen, H.; Winther, O. DeepLoc: prediction of protein subcellular localization using deep learning. Bioinformatics 2017, 33, 3387-3395.

74. Horton, P.; Park, K.J.; Obayashi, T.; Fujita, N.; Harada, H.; Adams-Collier, C.J.; Nakai, K. WoLF PSORT: protein localization predictor. Nucleic Acids Res. 2007, 35, W585-W587.

75. Emanuelsson, O.; Nielsen, H.; Brunak, S.; Von Heijne, G. Predicting subcellular localization of proteins based on their N-terminal amino acid sequence. J. mol. Biol. 2000, 300, 1005-1016.

76. Gasteiger, E.; Hoogland, C.; Gattiker, A.; Wilkins, M.R.; Appel, R.D.; Bairoch, A. Protein identification and analysis tools on the ExPASy server. In The proteomics protocols handbook; Humana press; 2005, 571-607.

77. Ferrè, F.; Clote, P. DiANNA: a web server for disulfide connectivity prediction. Nucleic Acids Res. 2005, 33, W230-W232.

78. Nakagawa, T.; Suzuki, T.; Murata, S.; Nakamura, S.; Hino, T.; Maeo, K.; Tabata, R.; Kawai, T.; Tanaka, K.; Niwa, Y.; et al. Improved Gateway binary vectors: highperformance vectors for creation of fusion constructs in transgenic analysis of plants. Biosci Biotechnol Biochem. 2007, 71, 2095-2100. 